\title{
Generalized Neumann Expansion and Its Application in Stochastic Finite Element Methods
}

\author{
Xiangyu Wang, ${ }^{1}$ Song Cen, ${ }^{1,2,3}$ and Chenfeng $\mathrm{Li}^{4}$ \\ ${ }^{1}$ Department of Engineering Mechanics, School of Aerospace, Tsinghua University, Beijing 100084, China \\ ${ }^{2}$ High Performance Computing Center, School of Aerospace, Tsinghua University, Beijing 100084, China \\ ${ }^{3}$ Key Laboratory of Applied Mechanics, School of Aerospace, Tsinghua University, Beijing 100084, China \\ ${ }^{4}$ College of Engineering, Swansea University, Singleton Park, Swansea SA2 8PP, UK \\ Correspondence should be addressed to Song Cen; censong@tsinghua.edu.cn
}

Received 18 July 2013; Accepted 13 August 2013

Academic Editor: Zhiqiang Hu

Copyright (c) 2013 Xiangyu Wang et al. This is an open access article distributed under the Creative Commons Attribution License, which permits unrestricted use, distribution, and reproduction in any medium, provided the original work is properly cited.

\begin{abstract}
An acceleration technique, termed generalized Neumann expansion (GNE), is presented for evaluating the responses of uncertain systems. The GNE method, which solves stochastic linear algebraic equations arising in stochastic finite element analysis, is easy to implement and is of high efficiency. The convergence condition of the new method is studied, and a rigorous error estimator is proposed to evaluate the upper bound of the relative error of a given GNE solution. It is found that the third-order GNE solution is sufficient to achieve a good accuracy even when the variation of the source stochastic field is relatively high. The relationship between the GNE method, the perturbation method, and the standard Neumann expansion method is also discussed. Based on the links between these three methods, quantitative error estimations for the perturbation method and the standard Neumann method are obtained for the first time in the probability context.
\end{abstract}

\section{Introduction}

Over the past few decades, the stochastic finite element method (SFEM) has attracted increasing attention from both academia and industries. The objective of SFEM is to analyze uncertainty propagation in practical engineering systems that are affected by various random factors, for example, heterogeneous materials and seismic loading. The current research of SFEM has two main focuses: probabilistic modeling of random media and efficient numerical solution of stochastic equations. Properties of heterogeneous materials (e.g., rocks and composites) vary randomly through the medium and are generally modeled with stochastic fields. Various probabilistic methods [1-7] have been developed to quantitatively describe the random material properties, which include the spectral representation method [1, 2], the KarhunenLoève expansion method $[3,4]$, and the Fourier-KarhunenLoève expansion method $[5,6]$, to name a few. In these methods, the random material properties are represented by a linear expansion such that they can be directly inserted into the governing equations of the physical system, which facilitates further numerical analysis. The resulting equations are stochastic equations, whose solution is the second main focus of SFEM research and also the focus of this work.

For static and steady-state problems, the resulting SFEM equations have the following form [8-13]:

$$
\left(\mathbf{A}_{0}+\mathbf{A}_{1} \alpha_{1}+\mathbf{A}_{2} \alpha_{2}+\cdots+\mathbf{A}_{m} \alpha_{m}\right) \mathbf{u}(\omega)=\mathbf{f}(\omega),
$$

where $\mathbf{A}_{1}, \ldots, \mathbf{A}_{m}$ are $n \times n$ symmetric and deterministic matrices, $\mathbf{u}(\omega)$ the unknown stochastic nodal vector, and $\mathbf{f}(\omega)$ the nodal force vector. If the material properties are modeled as Gaussian fields, then $\alpha_{1}, \alpha_{2}, \ldots, \alpha_{m}$ are independent Gaussian random variables. The uncertainties from the right-hand side of (1) are relatively easy to handle because they are not directly coupled with the random matrix from the left-hand side.

A number of methods have been developed for the solution of (1), including Monte Carlo methods, the perturbation method, the Neumann expansion method, and various projection methods. The Monte Carlo methods [1419] first generate a number of samples of the random material properties, then solve each sample with the deterministic 
FEM solver, and finally evaluate the statistics from these deterministic solutions. The Monte Carlo methods are simple, flexible, friendly to parallel computing, and not restricted by the number of random variables [20]. The computational cost of Monte Carlo methods is generally high especially for large-scale problems, although there exist a number of efficient sampling techniques such as important sampling $[14,19]$, subset simulation $[15,17]$, and line sampling $[18,19]$. Owning to its generosity and accuracy when sufficient sampling can be ensured, the Monte Carlo method is often taken as the reference method to verify other SFEM techniques. The perturbation method [11, 21-25] expresses the stiffness matrix, the load vector, and the unknown nodal displacement vector in terms of Taylor expansion with respect to the input random variables, and the Taylor expansion coefficients corresponding to the unknown nodal displacement are then solved by equating terms of the same order from both sides of (1). Its accuracy decreases when the random fluctuation increases, and there has not been a quantitative conclusion for the relationship between the solution error and the level of perturbation, making it difficult to confidently deploy the perturbation method in practical problems. The Neumann expansion method $[9,26,27]$ is an iterative process for the solution of linear algebraic equations. Because the spectral radius of the iterative matrix must be smaller than one, it is often considered not suitable for problems with large random variations. However, the fluctuation range allowed by the Neumann expansion method is generally higher than the perturbation methods, because it is easier to adopt higher order expansions in the former one. It should be noted that the stationary iterative procedure employed in Neumann expansion is not as efficient as the preconditioned conjugate gradient procedure employed in Monte Carlo methods. Therefore, the computational efficiency of the Neumann expansion method is inferior to direct Monte Carlo simulations. In the projection methods $[3,4,12,28-$ $35]$, the unknown nodal displacements are projected onto a set of orthogonal polynomials or other orthogonal bases, after which the Galerkin approach is employed to solve the projection coefficients. However, the projection methods typically result in an equation system much larger than the deterministic counterpart, and thus these methods are often limited to cases with a small set of base functions and a few independent random variables. In order to handle problems with more random variables, the stochastic collocation methods [36-42] have been developed, in which a reduced random basis is used in the projection operation and the projection coefficients are determined by approximating the precomputed Monte Carlo solutions. However, the stochastic collocation methods are believed to be less rigorous and their accuracy is poorer than the full projection methods using the Galerkin approach. Other variants of the projection procedures can be found in $[13,43,44]$. The fatal drawback of the projection types of solvers is their low efficiency [45], because the size of the base function set grows exponentially with the number of random variables and the order of the polynomials used. Moreover, the accuracy of the projection methods is criticized by $[46,47]$. For linear stochastic problems, a joint diagonalization solution strategy was proposed in [8-10], in which the stochastic solution is obtained in a closed form by jointly diagonalizing the matrix family in (1). This method is not sensitive to the number of random variables in the system, while there has not been a mathematically rigorous proof for its accuracy.

We propose a generalized Neumann expansion method for the solution of (1). The new GNE method is many times more efficient than the standard Neumann method, which is compromised by increased memory demand. In the context of probability, a mathematically rigorous error estimation is established for the new GNE scheme. It is also proved that for (1), the GNE scheme, the standard Neumann expansion method, and the perturbation method are equivalent. Hence, the stochastic error estimation obtained here also shed a light on the standard Neumann method and the perturbation method, which has remained as an outstanding challenge in the literature. The rest of the paper is organized as follows. The GNE scheme and its error estimation are derived in Section 2 , after which it is validated and examined in Section 3 via a comprehensive numerical test. Concluding remarks are given in Section 4.

\section{Generalized Neumann Expansion Method}

Consider a linear elastic statics system with Young's modulus modeled as a random field. By using the Karhunen-Loève expansion [3, 4] or the Fourier-Karhunen-Loève expansion $[5,6]$, random Young's modulus can be expressed as

$$
E(\mathbf{x}, \omega)=E_{0}(\mathbf{x})+\sum_{i} \sqrt{\lambda_{i}} \alpha_{i}(\omega) \psi_{i}(\mathbf{x}),
$$

where $E(\mathbf{x}, \omega)$ denotes random Young's modulus at point $\mathbf{x}$, $E_{0}(\mathbf{x})$ is the expectation of $E(\mathbf{x}, \omega), \alpha_{i}(\omega)$ are uncorrelated random variables, and $\lambda_{i}$ and $\psi_{i}(\mathbf{x})$ are deterministic eigen values and eigen functions corresponding to the covariance function of the source field $E(\mathbf{x}, \omega)$. If $E(\mathbf{x}, \omega)$ is modeled as a Gaussian field, $\alpha_{i}(\omega)$ become independent standard Gaussian random variables. For the sake of simplicity, we drop the random symbol $\omega$ and for the rest of the paper use $\alpha_{i}$ to denote the random variables in (2).

Following the standard finite element (FE) discretization procedure, a stochastic system of linear algebraic equations can be built from (2)

$$
\left(\mathbf{A}_{0}+\sum_{i} \alpha_{i} \mathbf{A}_{i}\right) \mathbf{u}=\mathbf{f} .
$$

In the above equation, $\mathbf{A}_{0}$ is the conventional FE stiffness matrix, which is symmetric, positive definite, and sparse while $\mathbf{A}_{i}$ are symmetric and sparse matrices. The deterministic matrices $\mathbf{A}_{i}$ share the same entry pattern as the stiffness matrix $\mathbf{A}_{0}$, but they are usually not positive definite.

2.1. Related Methods. The Monte Carlo methods first generate a set of samples of $\alpha_{i}$ in (3), and each sample of $\alpha_{i}$ corresponds to a deterministic equation, which can be solved by the standard FEM method. 
In the perturbation procedure, the unknown random vector $\mathbf{u}$ is expanded as $[21,25,26]$

$$
\mathbf{u}=\mathbf{u}_{0}+\sum_{i} \alpha_{i} \mathbf{u}_{i}+\sum_{i} \sum_{j} \alpha_{i} \alpha_{j} \mathbf{u}_{i j}+\cdots
$$

Substituting (4) into (3) yields

$$
\left(\mathbf{A}_{0}+\sum_{i} \alpha_{i} \mathbf{A}_{i}\right)\left(\mathbf{u}_{0}+\sum_{i} \alpha_{i} \mathbf{u}_{i}+\sum_{i} \sum_{j} \alpha_{i} \alpha_{j} \mathbf{u}_{i j}+\cdots\right)=\mathbf{f},
$$

from which the components $\mathbf{u}_{0}, \mathbf{u}_{i}, \mathbf{u}_{i j}$ of $\mathbf{u}$ are solved as follows:

$$
\begin{gathered}
\mathbf{u}_{0}=\mathbf{A}_{0}^{-1} \mathbf{f}, \\
\mathbf{u}_{i}=-\mathbf{A}_{0}^{-1} \mathbf{A}_{i} \mathbf{u}_{0}, \\
\mathbf{u}_{i j}=\mathbf{A}_{0}^{-1} \mathbf{A}_{i} \mathbf{A}_{0}^{-1} \mathbf{A}_{j} \mathbf{u}_{0} .
\end{gathered}
$$

It should be noted that, in the stochastic context, there has not been a rigorous convergence criterion for the perturbation scheme described above.

In the Neumann expansion method, the solution of (3) is written as

$$
\mathbf{u}=\left(\mathbf{A}_{0}+\Delta \mathbf{A}\right)^{-1} \mathbf{f}=(\mathbf{I}+\mathbf{B})^{-1} \mathbf{A}_{0}^{-1} \mathbf{f}
$$

where $\Delta \mathbf{A}=\sum_{i} \alpha_{i} \mathbf{A}_{i}, \mathbf{B}=\mathbf{A}_{0}^{-1} \Delta \mathbf{A}$ and $\mathbf{I}$ is the identity matrix. According to Neumann expansion

$$
(\mathbf{I}+\mathbf{B})^{-1}=\mathbf{I}-\mathbf{B}+\mathbf{B}^{2}-\mathbf{B}^{3}+\cdots,
$$

solution (7) can be simplified as

$$
\mathbf{u}=\mathbf{u}_{0}-\mathbf{B} \mathbf{u}_{0}+\mathbf{B}^{2} \mathbf{u}_{0}-\mathbf{B}^{3} \mathbf{u}_{0}+\cdots
$$

where $\mathbf{u}_{0}=\mathbf{A}_{0}^{-1} \mathbf{f}$. The spectral radius of matrix $\mathbf{B}$ must be smaller than 1 to ensure the convergence of (9).

2.2. The Generalized Neumann Expansion Method. The Neumann expansion (8) can be generalized to the inverse of multiple matrices:

$$
\left(\mathbf{I}+\sum_{i} \alpha_{i} \mathbf{B}_{i}\right)^{-1}=\mathbf{I}-\sum_{i} \alpha_{i} \mathbf{B}_{i}+\sum_{i} \sum_{j} \alpha_{i} \alpha_{j} \mathbf{B}_{i} \mathbf{B}_{j}-\cdots .
$$

The generalized Neumann expansion (GNE) in (10) can be proved as follows:

$$
\begin{gathered}
\left(\mathbf{I}+\sum_{i} \alpha_{i} \mathbf{B}_{i}\right)\left(\mathbf{I}+\sum_{i} \alpha_{i} \mathbf{B}_{i}\right)^{-1} \\
=\mathbf{I}-\sum_{i} \alpha_{i} \mathbf{B}_{i}+\sum_{i} \sum_{j} \alpha_{i} \alpha_{j} \mathbf{B}_{i} \mathbf{B}_{j}-\cdots \\
+\alpha_{1} \mathbf{B}_{1}-\sum_{i} \alpha_{1} \alpha_{i} \mathbf{B}_{1} \mathbf{B}_{i}+\cdots \\
+\alpha_{2} \mathbf{B}_{2}-\sum_{i} \alpha_{2} \alpha_{i} \mathbf{B}_{2} \mathbf{B}_{i}+\cdots
\end{gathered}
$$

$$
+\alpha_{n} \mathbf{B}_{n}-\sum_{i} \alpha_{n} \alpha_{i} \mathbf{B}_{n} \mathbf{B}_{i}+\cdots
$$

$=\mathbf{I}$,

where $n$ is the total number of $\mathbf{B}_{i}$.

The Neumann expansion (8) and the generalized Neumann expansion (10) are equivalent in mathematics and only differ in organizing the matrix operations. This difference can lead to a significant acceleration in computing the solution of (3). Specifically, let $\mathbf{B}_{i}=\mathbf{A}_{0}^{-1} \mathbf{A}_{i}$, and applying the GNE in (10) to (3) gives

$$
\begin{aligned}
\mathbf{u} & =\left(\mathbf{A}_{0}+\sum_{i} \alpha_{i} \mathbf{A}_{i}\right)^{-1} \mathbf{f}=\left(\mathbf{I}+\sum_{i} \alpha_{i} \mathbf{A}_{0}^{-1} \mathbf{A}_{i}\right)^{-1} \mathbf{A}_{0}^{-1} \mathbf{f} \\
& =\mathbf{u}_{0}-\sum_{i} \alpha_{i} \mathbf{A}_{0}^{-1} \mathbf{A}_{i} \mathbf{u}_{0}+\sum_{i} \sum_{j} \alpha_{j} \alpha_{i} \mathbf{A}_{0}^{-1} \mathbf{A}_{j} \mathbf{A}_{0}^{-1} \mathbf{A}_{i} \mathbf{u}_{0}-\cdots \\
& =\mathbf{u}_{0}-\sum_{i} \alpha_{i} \mathbf{u}_{i}+\sum_{i} \sum_{j} \alpha_{i} \alpha_{j} \mathbf{u}_{i j}-\cdots
\end{aligned}
$$

in which

$$
\begin{gathered}
\mathbf{u}_{i}=\mathbf{A}_{0}^{-1} \mathbf{A}_{i} \mathbf{u}_{0}, \\
\mathbf{u}_{i j}=\mathbf{A}_{0}^{-1} \mathbf{A}_{i} \mathbf{A}_{0}^{-1} \mathbf{A}_{j} \mathbf{u}_{0} .
\end{gathered}
$$

Equation (12) is the GNE solution.

2.3. Convergence Analysis and Error Estimation. The convergence condition of the GNE (10) is essentially the same as the Neumann expansion (8), that is, $\rho\left(\sum \alpha_{i} \mathbf{B}_{i}\right)<1$, where $\rho(\cdot)$ denotes the spectral radius of a matrix. Hence, for the GNE solution in (12), the convergence condition is

$$
\rho\left(\sum \alpha_{i} \mathbf{A}_{0}^{-1} \mathbf{A}_{i}\right)<1 .
$$

It should be noted that

$$
\rho\left(\sum \alpha_{i} \mathbf{A}_{0}^{-1} \mathbf{A}_{i}\right)=\rho\left(\sum \alpha_{i} \mathbf{C}^{-1} \mathbf{A}_{i} \mathbf{C}^{-1}\right)=\left\|\sum \alpha_{i} \mathbf{C}^{-1} \mathbf{A}_{i} \mathbf{C}^{-1}\right\|_{m_{2}},
$$


where $\|\cdot\|_{m_{2}}$ denotes the spectral norm of a matrix, and the symmetric and positive definite matrix $\mathbf{C}$ is defined as

$$
\mathbf{A}_{0}=\mathbf{C}^{2} \text {. }
$$

As a type of matrix norm, the spectral norm has the following properties:

$$
\begin{gathered}
\|\mathbf{P}+\mathbf{Q}\|_{m_{2}} \leq\|\mathbf{P}\|_{m_{2}}+\|\mathbf{Q}\|_{m_{2}}, \\
\|\mathbf{P Q}\|_{m_{2}} \leq\|\mathbf{P}\|_{m_{2}}\|\mathbf{Q}\|_{m_{2}}, \\
\|h \mathbf{P}\|_{m_{2}}=h\|\mathbf{P}\|_{m_{2}},
\end{gathered}
$$

where $\mathbf{P}$ and $\mathbf{Q}$ are general matrices and $h$ is a positive constant. Hence,

$$
\begin{aligned}
& \left\|\sum \alpha_{i} \mathbf{C}^{-1} \mathbf{A}_{i} \mathbf{C}^{-1}\right\|_{m_{2}} \\
& \leq\left\|\alpha_{1} \mathbf{C}^{-1} \mathbf{A}_{1} \mathbf{C}^{-1}\right\|_{m_{2}}+\left\|\alpha_{2} \mathbf{C}^{-1} \mathbf{A}_{2} \mathbf{C}^{-1}\right\|_{m_{2}} \\
& +\cdots\left\|\alpha_{n} \mathbf{C}^{-1} \mathbf{A}_{n} \mathbf{C}^{-1}\right\|_{m_{2}}, \\
& \rho\left(\sum \alpha_{i} \mathbf{C}^{-1} \mathbf{A}_{i} \mathbf{C}^{-1}\right) \\
& \leq \rho\left(\alpha_{1} \mathbf{C}^{-1} \mathbf{A}_{1} \mathbf{C}^{-1}\right)+\rho\left(\alpha_{2} \mathbf{C}^{-1} \mathbf{A}_{2} \mathbf{C}^{-1}\right) \\
& \quad+\cdots \rho\left(\alpha_{n} \mathbf{C}^{-1} \mathbf{A}_{n} \mathbf{C}^{-1}\right) \\
& \rho\left(\sum \alpha_{i} \mathbf{A}_{0}^{-1} \mathbf{A}_{i}\right) \\
& \leq \rho\left(\alpha_{1} \mathbf{A}_{0}^{-1} \mathbf{A}_{1}\right)+\rho\left(\alpha_{2} \mathbf{A}_{0}^{-1} \mathbf{A}_{2}\right)+\cdots \rho\left(\alpha_{n} \mathbf{A}_{0}^{-1} \mathbf{A}_{n}\right) \\
& \leq n \max \left(\alpha_{i}\right) \max \left(\rho\left(\mathbf{A}_{0}^{-1} \mathbf{A}_{i}\right)\right) .
\end{aligned}
$$

Based on the above relation, a sufficient but not necessary condition for convergence is

$$
n \max \left(\alpha_{i}\right) \max \left(\rho\left(\mathbf{A}_{0}^{-1} \mathbf{A}_{i}\right)\right)<1 .
$$

The maximum spectral radius $\rho_{\max }=\max \left(\rho\left(\mathbf{A}_{0}^{-1} \mathbf{A}_{i}\right)\right)$ can be obtained from $\mathbf{A}_{0}$ and $\mathbf{A}_{i}$. Equation (19) can be used to verify the convergence of GNE in advance. When $\alpha_{i}$ are independent standard Gaussian random variables, their absolute values are smaller than 3 with the probability of 99.7\%. In this case, (19) can be further simplified:

$$
\begin{gathered}
n \max \left(\alpha_{i}\right) \max \left(\rho\left(\mathbf{A}_{0}^{-1} \mathbf{A}_{i}\right)\right) \approx 3 n \rho_{\max }<1, \\
\rho_{\max }<\frac{1}{3 n} .
\end{gathered}
$$

Considering the $k$ th-order GNE (10) or Neumann expansion (8), their error can be estimated as follows:

$$
\begin{gathered}
\left\|(\mathbf{I}+\mathbf{B})^{-1}-\left(\mathbf{I}-\mathbf{B}+\mathbf{B}^{2}+\cdots+(-1)^{k} \mathbf{B}^{k}\right)\right\|_{m_{2}} \\
=\left\|\mathbf{B}^{k+1}(\mathbf{I}+\mathbf{B})^{-1}\right\|_{m_{2}} \\
\leq\left\|\mathbf{B}^{k+1}\right\|_{m_{2}}\left\|(\mathbf{I}+\mathbf{B})^{-1}\right\|_{m_{2}}=\frac{\sigma_{B}^{k+1}}{1-\sigma_{B}},
\end{gathered}
$$

where $\sigma_{B}$ is the spectral norm of $\mathbf{B}$, which is assumed to satisfy

$$
\sigma_{B}<1
$$

The exact solution of (3) is

$$
\begin{aligned}
\mathbf{u}=\left(\mathbf{I}+\sum_{i} \alpha_{i} \mathbf{A}_{0}^{-1} \mathbf{A}_{i}\right)^{-1} \mathbf{A}_{0}^{-1} \mathbf{f}=(\mathbf{I}+\mathbf{B})^{-1} \mathbf{A}_{0}^{-1} \mathbf{f}, \\
\mathbf{C u}=\left(\mathbf{I}+\sum_{i} \alpha_{i} \mathbf{C}^{-1} \mathbf{A}_{i} \mathbf{C}^{-1}\right)^{-1} \mathbf{C}^{-1} \mathbf{f} \\
=(\mathbf{I}+\mathbf{D})^{-1} \mathbf{C}^{-1} \mathbf{f},
\end{aligned}
$$

where $\mathbf{B}=\sum_{i} \alpha_{i} \mathbf{A}_{0}^{-1} \mathbf{A}_{i}, \mathbf{D}=\sum_{i} \alpha_{i} \mathbf{C}^{-1} \mathbf{A}_{i} \mathbf{C}^{-1}$. The $k$ th-order GNE or Neumann expansion solution is given by

$$
\begin{aligned}
\widetilde{\mathbf{u}} & =\left(\mathbf{I}-\mathbf{B}+\mathbf{B}^{2}+\cdots+(-1)^{k} \mathbf{B}^{k}\right) \mathbf{A}_{0}^{-1} \mathbf{f}, \\
\mathbf{C} \widetilde{\mathbf{u}} & =\left(\mathbf{I}-\mathbf{D}+\mathbf{D}^{2}+\cdots+(-1)^{k} \mathbf{D}^{k}\right) \mathbf{C}^{-1} \mathbf{f} .
\end{aligned}
$$

The norm of the solution error is defined as

$$
\begin{aligned}
\| \mathbf{C u} & -\mathbf{C} \widetilde{\mathbf{u}} \|_{2} \\
& =\left\|\left[(\mathbf{I}+\mathbf{D})^{-1}-\left(\mathbf{I}-\mathbf{D}+\mathbf{D}^{2}+\cdots+(-1)^{k} \mathbf{D}^{k}\right)\right] \mathbf{C}^{-1} \mathbf{f}\right\|_{2} \\
& =\left\|\mathbf{D}^{k+1}(\mathbf{I}+\mathbf{D})^{-1} \mathbf{C}^{-1} \mathbf{f}\right\|_{2}=\left\|\mathbf{D}^{k+1} \mathbf{C u}\right\|_{2} \\
& \leq\left\|\mathbf{D}^{k+1}\right\|_{m_{2}}\|\mathbf{C u}\|_{2}=\sigma_{D}^{k+1}\|\mathbf{C u}\|_{2}=\rho_{B}^{k+1}\|\mathbf{C u}\|_{2},
\end{aligned}
$$

where $\rho_{B}$ is the spectral radius of $\mathbf{B}$.

The relative error of the solution is defined as

$$
\begin{aligned}
\mathrm{RE} & =\frac{\sqrt{(\mathbf{u}-\widetilde{\mathbf{u}})^{T} \mathbf{A}_{0}(\mathbf{u}-\widetilde{\mathbf{u}})}}{\sqrt{\mathbf{u}^{T} \mathbf{A}_{0} \mathbf{u}}} \\
& =\frac{\sqrt{(\mathbf{u}-\widetilde{\mathbf{u}})^{T} \mathbf{C}^{2}(\mathbf{u}-\widetilde{\mathbf{u}})}}{\sqrt{\mathbf{u}^{T} \mathbf{C}^{2} \mathbf{u}}}=\frac{\|\mathbf{C u}-\mathbf{C} \widetilde{\mathbf{u}}\|_{2}}{\|\mathbf{C u}\|_{2}} \leq \rho_{B}^{k+1} .
\end{aligned}
$$

Comparing (21) and (26), it can be seen that the accuracy for the solution vector $\mathbf{u}$ is even better than the accuracy for the matrix inverse $(\mathbf{I}+\mathbf{B})^{-1}$.

2.4. Solutions for More General Stochastic Algebraic Equations. For the solution of (3), the GNE solution in (12) shares the same form as the perturbation solution in (5). Hence, for solving (3), the GNE method, the perturbation method, and the standard Neumann expansion method are identical. The error estimation obtained in (26) also holds for the perturbation approach.

However, for the following more general stochastic algebraic equations [26]:

$$
\begin{aligned}
\left(\mathbf{A}_{0}\right. & \left.+\sum_{i} \alpha_{i} \mathbf{A}_{i}+\sum_{i} \sum_{j} \alpha_{i} \alpha_{j} \mathbf{A}_{i j}+\cdots\right) \mathbf{u} \\
& =\mathbf{f}_{0}+\sum_{i} \gamma_{i} \mathbf{f}_{i}+\sum_{i} \sum_{j} \gamma_{i} \gamma_{j} \mathbf{f}_{i j}+\cdots,
\end{aligned}
$$


the three methods are not equivalent. Specifically, applying the perturbation method to the above equation yields

$$
\left(\mathbf{A}_{0}+\sum_{i} \alpha_{i} \mathbf{A}_{i}+\sum_{i} \sum_{j} \alpha_{i} \alpha_{j} \mathbf{A}_{i j}+\cdots\right)
$$

Equation (28) can be reorganized as

$$
\begin{aligned}
& \mathbf{A}_{0} \mathbf{u}_{0} \quad+\sum_{i} \alpha_{i} \mathbf{A}_{i} \mathbf{u}_{0} \quad+\sum_{i} \sum_{j} \alpha_{i} \alpha_{j} \mathbf{A}_{i j} \mathbf{u}_{0} \quad \ldots \\
& +\sum_{i} \mathbf{A}_{0} \beta_{i} \mathbf{u}_{i} \quad+\sum_{i} \sum_{j}^{i} \alpha_{i} \mathbf{A}_{i} \beta_{j} \mathbf{u}_{j} \quad+\sum_{i} \sum_{j}^{i} \sum_{k}^{j} \alpha_{i} \alpha_{j} \mathbf{A}_{i j} \beta_{k} \mathbf{u}_{k} \\
& +\sum_{i} \sum_{j}^{i} \mathbf{A}_{0} \beta_{i} \beta_{j} \mathbf{u}_{i j}+\sum_{i} \sum_{j} \sum_{k}^{j} \alpha_{i} \mathbf{A}_{i} \beta_{j} \beta_{k} \mathbf{u}_{j k}+\sum_{i} \sum_{j} \sum_{k} \sum_{l}^{k} \alpha_{i} \alpha_{j} \mathbf{A}_{i j} \beta_{k} \beta_{l} \mathbf{u}_{k l} \cdots \\
& =\mathbf{f}_{0}+\sum_{i} \gamma_{i} \mathbf{f}_{i}+\sum_{i} \sum_{j} \gamma_{i} \gamma_{j} \mathbf{f}_{i j}+\cdots
\end{aligned}
$$

If $\alpha_{i}=\beta_{i}=\gamma_{i}$, then the perturbation components $\mathbf{u}_{0}, \mathbf{u}_{i}, \mathbf{u}_{i j}$, $\ldots, \mathbf{u}_{i_{1} i_{2} \cdots i_{n}}$ of $\mathbf{u}$ can be solved as

$$
\begin{gathered}
\mathbf{A}_{0} \mathbf{u}_{0}=\mathbf{f}_{0}, \\
\mathbf{A}_{0} \mathbf{u}_{i}+\mathbf{A}_{i} \mathbf{u}_{0}=\mathbf{f}_{i}, \\
\mathbf{A}_{0} \mathbf{u}_{i j}+\mathbf{A}_{i} \mathbf{u}_{j}+\mathbf{A}_{i j} \mathbf{u}_{0}=\mathbf{f}_{i j}, \\
\vdots \\
\mathbf{A}_{0} \mathbf{u}_{i_{1} i_{2} \cdots i_{n}}+\mathbf{A}_{i_{1}} \mathbf{u}_{i_{2} i_{3} \cdots i_{n}}+\mathbf{A}_{i_{1} i_{2}} \mathbf{u}_{i_{3} i_{4} \cdots i_{n}} \\
+\mathbf{A}_{i_{1} i_{2} \cdots i_{n}} \mathbf{u}_{0}=\mathbf{f}_{i_{1} i_{2} \cdots i_{n}} .
\end{gathered}
$$

Solving (27) with the Neumann expansion gives

$$
\begin{aligned}
\mathbf{A}^{-1} & =\left(\mathbf{A}_{0}+\Delta \mathbf{A}\right)^{-1} \\
& =(\mathbf{I}+\mathbf{B})^{-1} \mathbf{A}_{0}^{-1}=\left(\mathbf{I}-\mathbf{B}+\mathbf{B}^{2}-\mathbf{B}^{3}+\cdots\right) \mathbf{A}_{0}^{-1},
\end{aligned}
$$

where $\mathbf{B}=\mathbf{A}_{0}^{-1} \Delta \mathbf{A}, \Delta \mathbf{A}=\sum_{i} \alpha_{i} \mathbf{A}_{i}+\sum_{i} \sum_{j} \alpha_{i} \alpha_{j} \mathbf{A}_{i j}+\cdots$. Then

$$
\mathbf{u}=\left(\mathbf{I}-\mathbf{B}+\mathbf{B}^{2}-\mathbf{B}^{3}+\cdots\right) \mathbf{A}_{0}^{-1}\left(\mathbf{f}_{0}+\Delta \mathbf{f}\right),
$$

in which

$$
\Delta \mathbf{f}=\sum_{i} \gamma_{i} \mathbf{f}_{i}+\sum_{i} \sum_{j} \gamma_{i} \gamma_{j} \mathbf{f}_{i j}+\cdots
$$

Because higher order entries are included in $\mathbf{B}$, the term $\mathbf{B A}_{0}^{-1}\left(\mathbf{f}_{0}+\Delta \mathbf{f}\right)$ (the first-order term of the Neumann solution in (32)) does not correspond to the first-order entries of $\mathbf{u}$. Therefore, the Neumann solution in (32) and the perturbation solution in (30) are different for the general stochastic equation (27).
Solving (27) with the GNE approach gives

$$
\begin{gathered}
\mathbf{u}=\left(\mathbf{A}_{0}+\sum_{i_{i}} \alpha_{i_{1}} \mathbf{A}_{i_{1}}+\sum_{i_{2}} \sum_{i_{1}} \alpha_{i_{1}} \alpha_{i_{2}} \mathbf{A}_{i_{1} i_{2}}+\cdots\right. \\
\left.+\sum_{i_{n}} \cdots \sum_{i_{2}} \sum_{i_{1}} \alpha_{i_{1} i_{2} \cdots i_{n}} \mathbf{A}_{i_{1} i_{2} \cdots i_{n}}\right)^{-1} \mathbf{f} \\
=\left(\mathbf{I}+\sum_{i_{i}} \alpha_{i_{1}} \mathbf{B}_{i_{1}}+\sum_{i_{2}} \sum_{i_{1}} \alpha_{i_{1}} \alpha_{i_{2}} \mathbf{B}_{i_{1} i_{2}}+\cdots\right. \\
\left.+\sum_{i_{n}} \cdots \sum_{i_{2}} \sum_{i_{1}} \alpha_{i_{1} i_{2} \cdots i_{n}} \mathbf{B}_{i_{1} i_{2} \cdots i_{n}}\right)^{-1} \mathbf{A}_{0}^{-1} \mathbf{f}
\end{gathered}
$$

$$
\begin{aligned}
= & \left(\mathbf{I}+\sum_{i} \beta_{i} \mathbf{C}_{i}\right)^{-1} \mathbf{A}_{0}^{-1} \mathbf{f} \\
= & \left(\mathbf{I}-\sum_{i} \beta_{i} \mathbf{C}_{i}+\sum_{i} \sum_{j} \beta_{i} \beta_{j} \mathbf{C}_{i} \mathbf{C}_{j}\right. \\
& \left.-\sum_{i} \sum_{j} \sum_{k} \beta_{i} \beta_{j} \beta_{k} \mathbf{C}_{i} \mathbf{C}_{j} \mathbf{C}_{k}+\cdots\right) \mathbf{A}_{0}^{-1} \mathbf{f},
\end{aligned}
$$

where $\mathbf{f}=\mathbf{f}_{0}+\sum_{i} \gamma_{i} \mathbf{f}_{i}+\sum_{i} \sum_{j} \gamma_{i} \gamma_{j} \mathbf{f}_{i j}+\cdots, \mathbf{B}_{i}=\mathbf{A}_{0}^{-1} \mathbf{A}_{i}$, $\mathbf{B}_{i j}=\mathbf{A}_{0}^{-1} \mathbf{A}_{i j}$, and other $\mathbf{B}$ terms can be similarly formed. Matrices $\mathbf{C}_{i}$ are determined by realignment of $\mathbf{B}_{i}, \mathbf{B}_{i j}, \ldots$ and random variables $\beta_{i}$ are formed by rearrangement of $\alpha_{i_{1}}, \alpha_{i_{1} i_{2}}, \ldots, \alpha_{i_{1} i_{2} \ldots i_{n}}$.

In summary, when solving (27), the GNE method and the Neumann method are identical, while they are not equivalent to the perturbation procedure. However, if the higher order terms in the GNE or Neumann expansion solutions are omitted, they degenerate into the perturbation solution. 


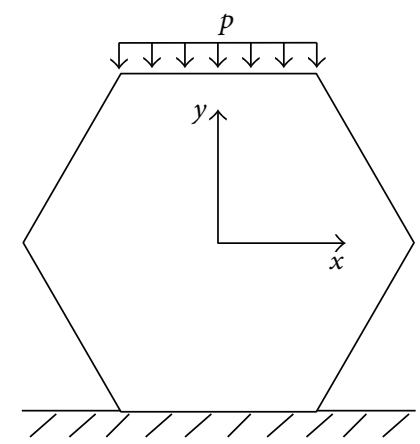

FIGURE 1: Cross section of the random material sample.

Two simplified examples are given here to demonstrate the difference between the GNE and the perturbation method.

2.4.1. Example I. Only first-order entries are included in both sides of (27) such that the equation becomes

$$
\left(\mathbf{A}_{0}+\sum_{i} \alpha_{i} \mathbf{A}_{i}\right) \mathbf{u}=\mathbf{f}_{0}+\sum_{i} \alpha_{i} \mathbf{f}_{i}
$$

The first-order perturbation solution is

$$
\begin{aligned}
\mathbf{u}_{0} & =\mathbf{A}_{0}^{-1} \mathbf{f}_{0}, \\
\mathbf{u}_{i} & =\mathbf{A}_{0}^{-1} \mathbf{f}_{i}-\mathbf{A}_{0}^{-1} \mathbf{A}_{i} \mathbf{u}_{0}, \\
\mathbf{u} & =\mathbf{u}_{0}+\sum_{i} \alpha_{i} \mathbf{u}_{i} \\
& =\mathbf{A}_{0}^{-1} \mathbf{f}_{0}+\sum_{i} \alpha_{i} \mathbf{A}_{0}^{-1} \mathbf{f}_{i}-\sum_{i} \alpha_{i} \mathbf{A}_{0}^{-1} \mathbf{A}_{i} \mathbf{u}_{0},
\end{aligned}
$$

and the first-order GNE solution is

$$
\begin{aligned}
\mathbf{u}= & \left(\mathbf{A}_{0}^{-1}-\sum_{i} \alpha_{i} \mathbf{A}_{0}^{-1} \mathbf{A}_{i} \mathbf{A}_{0}^{-1}\right)\left(\mathbf{f}_{0}+\sum_{i} \alpha_{i} \mathbf{f}_{i}\right) \\
= & \mathbf{A}_{0}^{-1} \mathbf{f}_{0}-\sum_{i} \alpha_{i} \mathbf{A}_{0}^{-1} \mathbf{A}_{i} \mathbf{u}_{0}+\sum_{i} \alpha_{i} \mathbf{A}_{0}^{-1} \mathbf{f}_{i} \\
& -\sum_{i} \sum_{j} \alpha_{i} \alpha_{j} \mathbf{A}_{0}^{-1} \mathbf{A}_{i} \mathbf{f}_{j} .
\end{aligned}
$$

Comparing (36) and (37), it can be seen that the GNE solution contains an extra second-order term $-\sum_{i} \sum_{j} \alpha_{i} \alpha_{j} \mathbf{A}_{0}^{-1} \mathbf{A}_{i} \mathbf{f}_{j}$.

2.4.2. Example II. Up to second-order terms are included in the left-hand side of (27), while only the constant term is included in the right-hand side. In this case, the equation becomes

$$
\left(\mathbf{A}_{0}+\sum_{i} \alpha_{i} \mathbf{A}_{i}+\sum_{i} \sum_{j} \alpha_{i} \alpha_{j} \mathbf{A}_{i j}\right) \mathbf{u}=\mathbf{f}_{0}
$$

Denote $\mathbf{u}_{0}=\mathbf{A}_{0}^{-1} \mathbf{f}_{0}, \mathbf{B}_{i}=\mathbf{A}_{0}^{-1} \mathbf{A}_{i}$, and $\mathbf{B}_{i j}=\mathbf{A}_{0}^{-1} \mathbf{A}_{i j}$; then the first-order perturbation solution can be expressed as

$$
\begin{gathered}
\mathbf{u}_{0}=\mathbf{A}_{0}^{-1} \mathbf{f}_{0}, \\
\mathbf{u}_{i}=-\sum_{i} \mathbf{A}_{0}^{-1} \mathbf{A}_{i} \mathbf{u}_{0}=-\sum_{i} \mathbf{B}_{i} \mathbf{u}_{0}, \\
\mathbf{u}=\mathbf{u}_{0}+\sum_{i} \alpha_{i} \mathbf{u}_{i}=\mathbf{u}_{0}-\sum_{i} \alpha_{i} \mathbf{B}_{i} \mathbf{u}_{0},
\end{gathered}
$$

while the first-order GNE solution is

$$
\begin{aligned}
\mathbf{u} & =\left(\mathbf{I}+\sum_{i} \alpha_{i} \mathbf{A}_{0}^{-1} \mathbf{A}_{i}+\sum_{i} \sum_{j} \alpha_{i} \alpha_{j} \mathbf{A}_{0}^{-1} \mathbf{A}_{i j}\right)^{-1} \mathbf{u}_{0} \\
& =\left(\mathbf{I}+\sum_{i} \alpha_{i} \mathbf{B}_{i}+\sum_{i} \sum_{j} \alpha_{i} \alpha_{j} \mathbf{B}_{i j}\right)^{-1} \mathbf{u}_{0} \\
& =\left(\mathbf{I}-\sum_{i} \alpha_{i} \mathbf{B}_{i}-\sum_{i} \sum_{j} \alpha_{i} \alpha_{j} \mathbf{B}_{i j}\right) \mathbf{u}_{0} .
\end{aligned}
$$

Comparing (39) and (40), the second-order terms $-\sum_{i} \sum_{j} \alpha_{i} \alpha_{j} \mathbf{A}_{0}^{-1} \mathbf{A}_{i j} \mathbf{u}_{0}$ in the GNE solution do not appear in the perturbation solution (39).

\section{Numerical Examples}

3.1. Example Description. As shown in Figure 1, a plain strain problem with an orthohexagonal cross section is considered here. The length of each edge of the hexagon is $0.4 \mathrm{~m}$. The Poisson ratio of the material is set as 0.2 , and Young's modulus is assumed to be a Gaussian field with a mean value of $30 \mathrm{GPa}$ and a covariance function defined as

$$
R\left(\tau_{1}, \tau_{2}\right)=c_{1} e^{-\tau_{1}^{2} / c_{2}-\tau_{2}^{2} / c_{2}},
$$

in which $\tau_{1}=x_{1}-x_{2}$ and $\tau_{2}=y_{1}-y_{2}$ are the coordinate differences and $c_{1}$ and $c_{2}$ are adjustable constants. As shown in Figure 2, the cross section is discretized with triangular linear elements, where each side is divided into 8 equal parts. There are in total 384 elements and 217 nodes. 


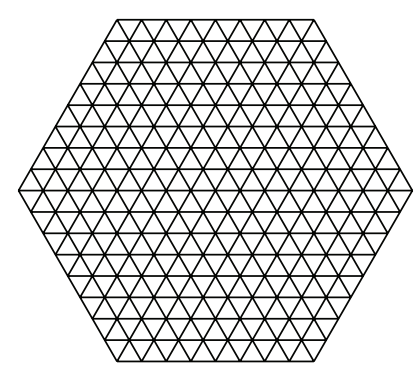

Figure 2: Finite element mesh.

The relative error is defined as

$$
\mathrm{RE}=\frac{\left\|\mathbf{C} \mathbf{u}_{\mathrm{GNE}}-\mathbf{C} \mathbf{u}_{\mathrm{MC}}\right\|_{2}}{\left\|\mathrm{C} \mathbf{u}_{\mathrm{MC}}\right\|_{2}} \times 100 \%,
$$

in which $\mathbf{u}_{\mathrm{MC}}$ is the nodal displacement vector solved with the Monte Carlo method and $\mathbf{u}_{\mathrm{GNE}}$ is the GNE solution. Unless stated explicitly, the numerical testing is performed for 4340 samples generated from the Gaussian field defined in (41).

The convergence condition of the GNE method has been rigorously proved. The main purpose of this example is to examine the error distribution and its relationship with various random parameters, for which a number of numerical tests are performed in the following subsections.

3.2. Error Estimation. The third-order GNE solution is adopted. The constants $c_{1}$ and $c_{2}$ in (41) are varied to examine the error estimator in (26). The constant $c_{1}$ is the variance of the Gaussian field, and the constant $c_{2}$ controls the shape of the covariance function. The effective correlation length $L_{\text {eff }}$ of the covariance function $R(\tau)$ is defined by

$$
\frac{R\left(L_{\mathrm{eff}}\right)}{R(0)}=0.01
$$

For any two points in the medium, if the distance between them is larger than $L_{\text {eff }}$, their material properties are only weakly correlated. The effective correlation length of the Gaussian field defined in (41) is

$$
\frac{R(\tau)}{R(0)}=e^{-\tau^{2} / c_{2}}=0.01 \Longrightarrow L_{\mathrm{eff}}=2.146 \sqrt{c_{2}} .
$$

In the first set of tests, the constant $c_{1}$ is varied to change the variance of the Gaussian field. The parameter settings are

$$
\begin{array}{lll}
c_{1}=0.01, & c_{2}=3.2 E-2, & L_{\text {eff }}=0.38, \\
c_{1}=0.04, & c_{2}=3.2 E-2, & L_{\text {eff }}=0.38 .
\end{array}
$$

In the second set of tests, the constant $c_{2}$ is varied to change the shape of the covariance function. The parameter settings are

$$
\begin{array}{lll}
c_{1}=0.01, & c_{2}=0.8 E-2, & L_{\mathrm{eff}}=0.19, \\
c_{1}=0.01, & c_{2}=2.4 E-2, & L_{\mathrm{eff}}=0.33, \\
c_{1}=0.01, & c_{2}=4.0 E-2, & L_{\mathrm{eff}}=0.43 .
\end{array}
$$

3.2.1. The Influence of $c_{1}$. Corresponding to $c_{1}=0.01$ and $c_{1}=0.04$, the standard deviation $\sigma$ of the stochastic field is 0.1 and 0.2 , respectively. A total number of 4340 samples are generated for each case. For each realization, both the Monte Carlo solution and the third-order GNE solution are computed. The relationship between the spectral radius and the relative error is drawn in Figures 3 and 4, in which the red crosses show the actual relative errors corresponding to each sample and the blue line shows the theoretical error estimation. It can been seen that for both cases, the theoretical error estimator in (26) holds accurately and its effectiveness is not affected by the change of the variance controlled by $c_{1}$.

3.2.2. The Influence of $c_{2}$. The constant $c_{2}$ is adjusted to take three different values $0.8 \times 10^{-2}, 2.4 \times 10^{-2}$, and $4.0 \times 10^{-2}$, while the constant $c_{1}$ remains unchanged. Following (41), the effective correlation length grows with the increase of $c_{2}$. Again, 4340 samples are generated for each stochastic field, and for each realization both the Monte Carlo solution and the third-order GNE solution are computed. Figure 5 plots the relationship between spectral radius and the relative error, which verifies that, for all cases, the theoretical error estimation in (26) holds. The changing of $c_{2}$, which changes the variation frequency of the stochastic field, does not affect the error estimation.

As indicated in (44), the effective correlation length $L_{\text {eff }}$ is directly associated with the parameter $c_{2}$. To investigate the influence of $L_{\text {eff }}$ on the relative error, the empirical cumulative distribution functions (ECDF) of relative errors are plotted in Figure 6 for different $L_{\text {eff }}$ cases. Comparing the three ECDF curves at different effective correlation lengths, it is found that with the decrease of the effective correlation length, the accuracy of the GNE solution improves.

3.3. The Relative Error for Other Types of Stochastic Fields. In the last two subsections, the error estimation analysis has taken the Gaussian assumption; that is, all random variables $\alpha_{i}$ in (3) are assumed to be independent Gaussian random variables. In this subsection, we investigate the relative error of the GNE method for other types of stochastic fields. Specifically, the random variables $\alpha_{i}$ in (3) are assumed to have the two-point distribution, the binomial distribution, and the $\beta$ distribution, respectively. For the case of two-point distribution, values $\left\{-0.3 E_{0}, 0.3 E_{0}\right\}$ are assigned with equal probability, that is, 0.5 . For the case of binomial distribution, ten times Bernoulli trials with the probability 0.5 are adopted, 


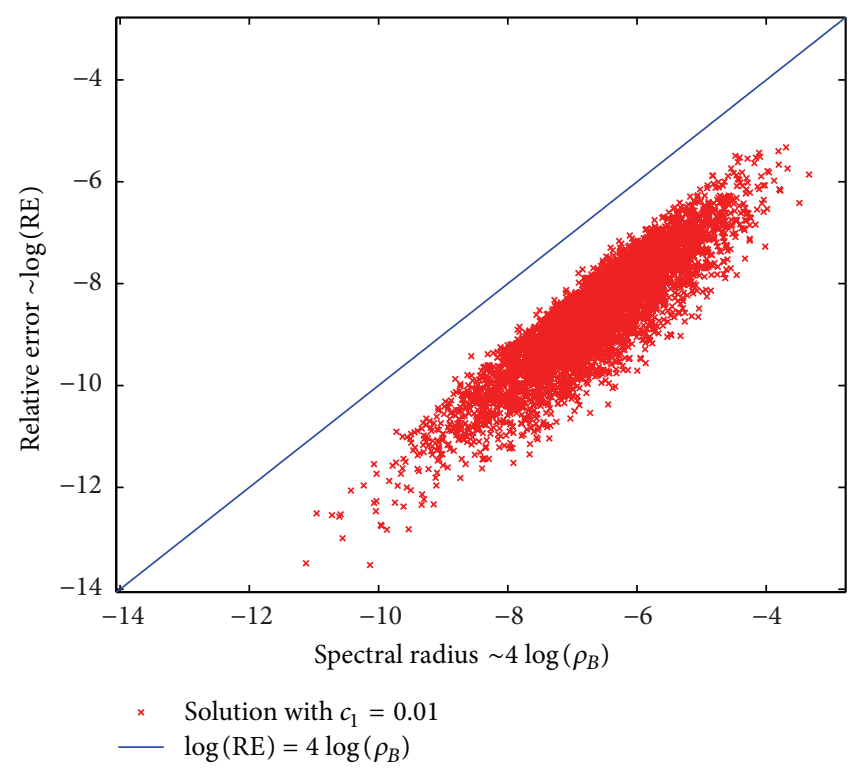

FIGURE 3: The relation between the relative error and the spectral radius.

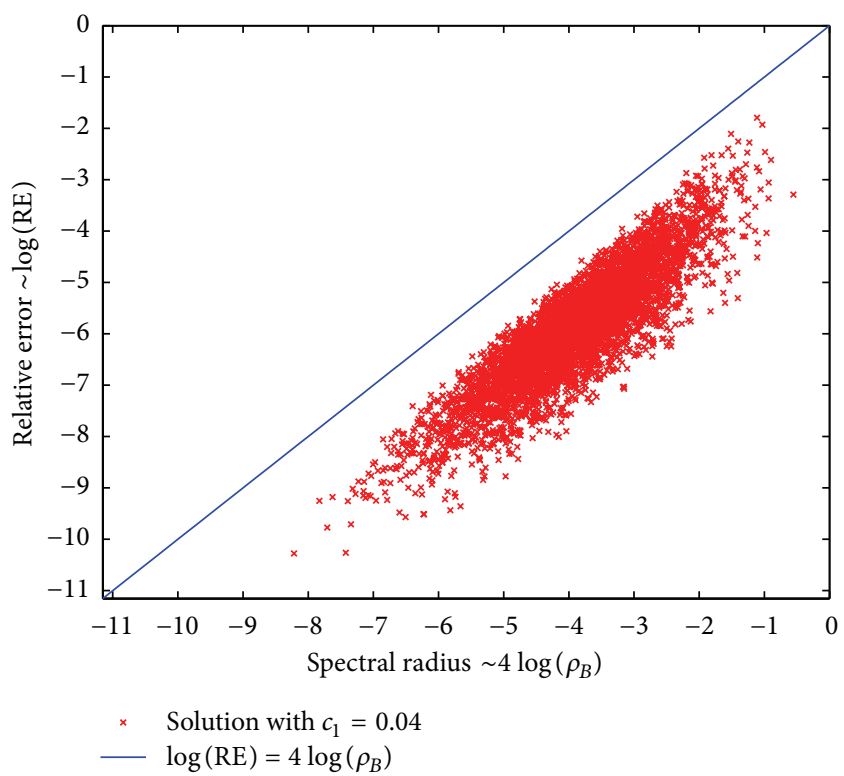

FIGURE 4: The relation between the relative error and the spectral radius.

and the integer values between 0 and 10 are linearly projected onto $\left[-0.3 E_{0}, 0.3 E_{0}\right]$. For the case of $\beta$ distribution, the probability density function is defined as

$$
f(x \mid a, b)=\frac{1}{B(a, b)} x^{a-1}(1-x)^{b-1} I_{(0,1)}(x),
$$

where $B(a, b)$ is a $\beta$ function, and the filter function $I_{(0,1)}(x)$ is defined as

$$
I_{(0,1)}(x)= \begin{cases}1 & x \in(0,1) \\ 0 & x \notin(0,1)\end{cases}
$$

Three kinds of $\beta$ distributions are considered, with $a=b=$ $0.75, a=b=1$, and $a=b=4$, respectively. The interval $(0,1)$ is linearly mapped to $\left[-0.3 E_{0}, 0.3 E_{0}\right]$.

Corresponding to these five distributions, the empirical cumulative distribution functions of relative errors are plotted in Figure 7. Comparing the ECDF curves at different effective correlation lengths, the same trend is observed as the Gaussian case. That is, the relative error decreases with the drop of effective correlation length.

3.4. Efficiency Analysis. Using the same computing platform, the Monte Carlo method, the Neumann expansion method, 


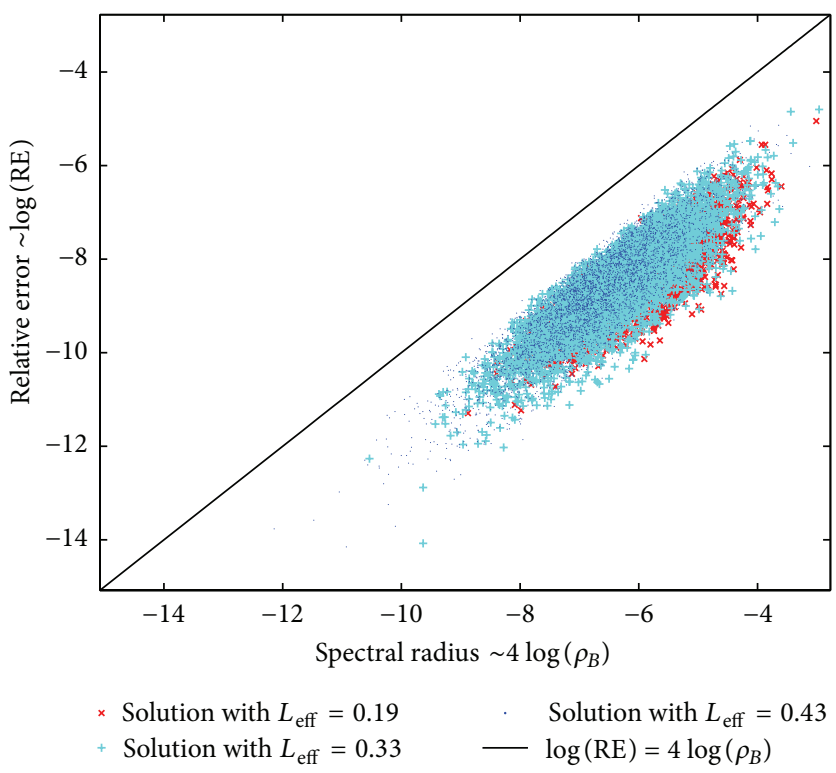

FiguRE 5: The relation between the relative error and the spectral radius.

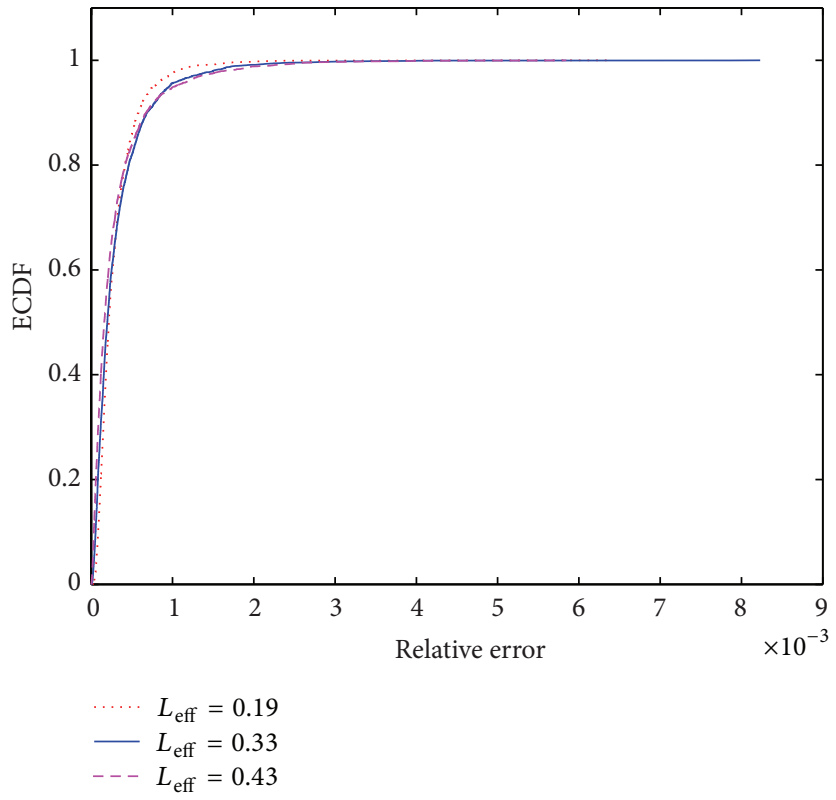

FIGURE 6: ECDF of the relative errors.

and the GNE method are employed to solve the same set of solutions (corresponding to 4340 samples), and the computation time is shown in Table 1 . The number of random variables in the stochastic equation system differs depending on the effective correlation length. A fixed mesh is adopted in all of the computation such that the dimension of a single equation system is $434 \times 434$.

If the mesh density is doubled, then the dimension of each matrix is $1634 \times 1634$. The number of random variables remains the same in each case. The corresponding computation time is shown in Table 2.
The two data tables indicate that the computational efficiency of the GNE method (up to the third order) is much higher than Neumann expansion and Monte Carlo procedures. However, the memory requirement for higher order GNE schemes increases rapidly causing the computational efficiency to decrease.

\section{Conclusions}

An acceleration approach of Neumann expansion is proposed for solving stochastic linear equations. The new method, 


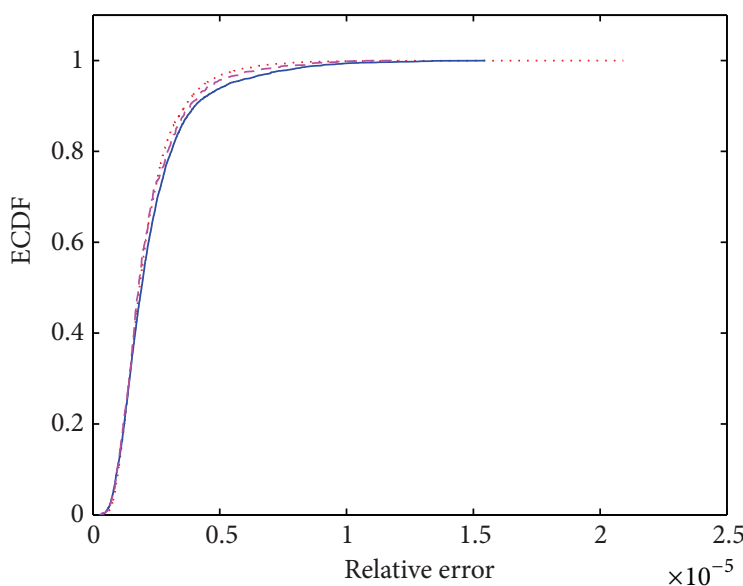

(a) Two-point distribution

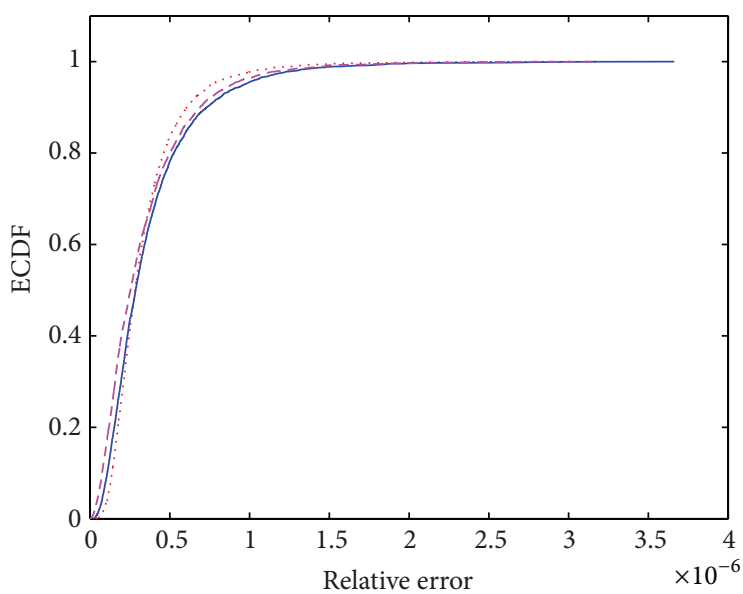

(c) $\beta$ distribution with $a=b=0.75$

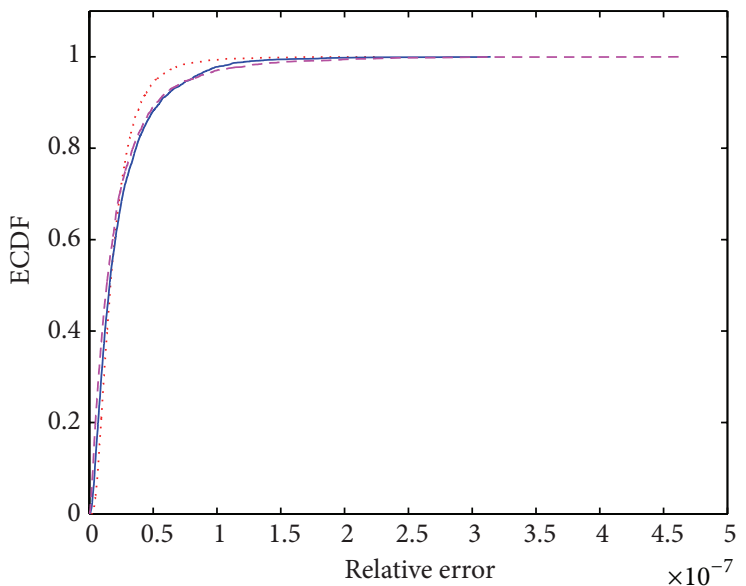

(b) Binomial distribution

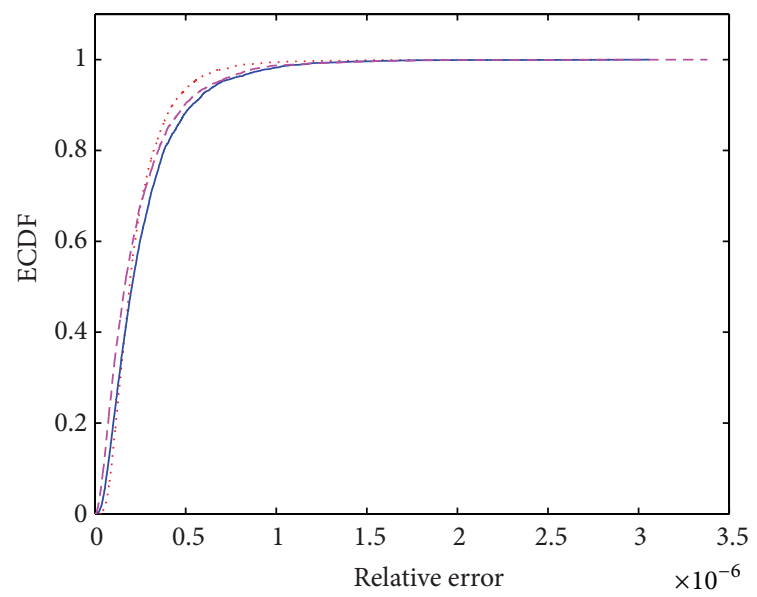

(d) $\beta$ distribution with $a=b=1$

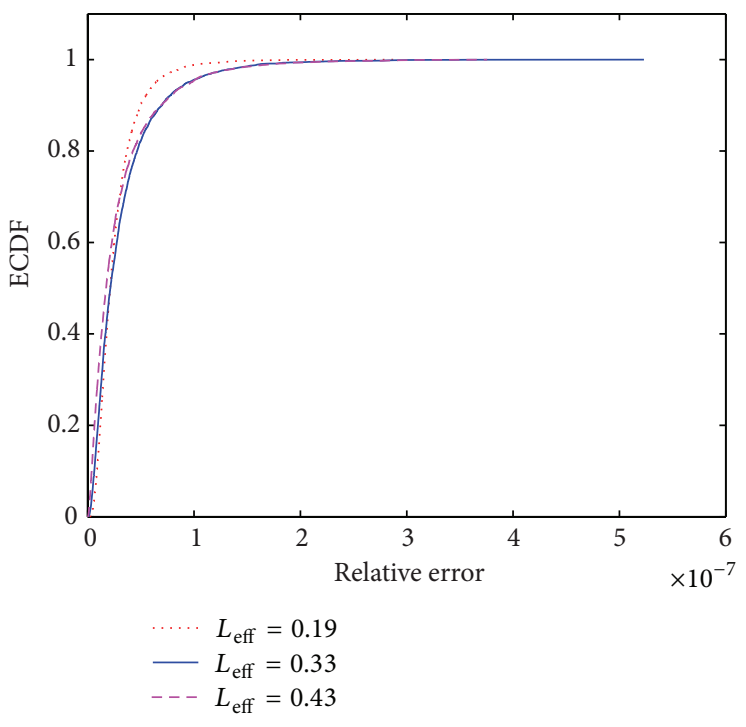

(e) $\beta$ distribution with $a=b=4$

FIGURE 7: ECDF of the relative error. 
TABLE 1: Computation time for 4340 samples (unit: s).

\begin{tabular}{|c|c|c|c|c|c|}
\hline Number of random variables & 10 & 11 & 16 & 21 & 39 \\
\hline Monte Carlo & 40.78 & 43.26 & 54.02 & 65.55 & 107.13 \\
\hline \multicolumn{6}{|l|}{ GNE } \\
\hline First order & 0.44 & 0.44 & 0.48 & 0.53 & 0.69 \\
\hline Second order & 0.79 & 0.83 & 1.05 & 1.28 & 2.21 \\
\hline Third order & 1.30 & 1.43 & 2.29 & 3.58 & 13.33 \\
\hline \multicolumn{6}{|l|}{ Neumann } \\
\hline First order & 26.74 & 28.96 & 40.43 & 52.23 & 93.90 \\
\hline Second order & 28.63 & 30.88 & 42.28 & 53.70 & 95.22 \\
\hline Third order & 30.28 & 32.57 & 44.05 & 55.49 & 97.08 \\
\hline
\end{tabular}

TABLE 2: Computation time for 16340 samples (unit: s).

\begin{tabular}{|c|c|c|c|}
\hline Number of random variables & 11 & 16 & 21 \\
\hline Monte Carlo & 6560.01 & 7871.31 & 9118.39 \\
\hline \multicolumn{4}{|l|}{ GNEM } \\
\hline First order & 13.31 & 14.85 & 16.39 \\
\hline Second order & 16.06 & 19.23 & 22.50 \\
\hline Third order & 24.55 & 41.86 & 70.97 \\
\hline \multicolumn{4}{|l|}{ Neumann } \\
\hline First order & 3325.30 & 4851.50 & 6017.91 \\
\hline Second order & 3639.38 & 5010.79 & 6314.89 \\
\hline Third order & 3881.43 & 5473.71 & 6609.92 \\
\hline
\end{tabular}

termed generalized Neumann expansion, generalizes Neumann expansion from one matrix to multiple matrices. The GNE method is equivalent to Neumann expansion for general stochastic equations, and for stochastic linear equations, both methods are also equivalent to the perturbation method. According to this equivalence relation, a rigorous error estimation is obtained, which is applicable to all the three methods. Numerical tests show that the third-order GNE expansion can provide good accuracy even for relatively large random fluctuations.

\section{Conflict of Interests}

The authors do not have any conflict of interests with the content of the paper.

\section{Acknowledgments}

The authors would like to acknowledge the financial supports of the National Natural Science Foundation of China (11272181), the Specialized Research Fund for the Doctoral Program of Higher Education of China (20120002110080), and the National Basic Research Program of China (Project no. 2010CB832701). The authors would also like to thank the support from British Council and Chinese Scholarship
Council through the support of an award for Sino-UK Higher Education Research Partnership for PhD Studies.

\section{References}

[1] M. Shinozuka and G. Deodatis, "Simulation of stochastic processes by spectral representation," Applied Mechanics Reviews, vol. 44, p. 191, 1991.

[2] M. Shinozuka and G. Deodatis, "Simulation of multi-dimensional Gaussian stochastic fields by spectral representation," Applied Mechanics Reviews, vol. 49, no. 1, pp. 29-53, 1996.

[3] R. G. Ghanem and P. D. Spanos, "Spectral techniques for stochastic finite elements," Archives of Computational Methods in Engineering, vol. 4, no. 1, pp. 63-100, 1997.

[4] R. G. Ghanem and P. D. Spanos, Stochastic Finite Elements: A spectral Approach, Courier Dover Publications, 2003.

[5] C. F. Li, Y. T. Feng, D. R. J. Owen, and I. M. Davies, "Fourier representation of random media fields in stochastic finite element modelling," Engineering Computations, vol. 23, no. 7, pp. 794-817, 2006.

[6] C. F. Li, Y. T. Feng, D. R. J. Owen, D. F. Li, and I. M. Davis, "A Fourier-Karhunen-Loève discretization scheme for stationary random material properties in SFEM," International Journal for Numerical Methods in Engineering, vol. 73, no. 13, pp. 1942-1965, 2008. 
[7] J. W. Feng, C. F. Li, S. Cen, and D. R. J. Owen, "Statistical reconstruction of two-phase random media," Computers \& Structures, 2013.

[8] C. F. Li, Y. T. Feng, and D. R. J. Owen, "Explicit solution to the stochastic system of linear algebraic equations $\left(\alpha_{1} A_{1}+\alpha_{2} A_{2}+\right.$ $\left.\cdots+\alpha_{m} A_{m}\right) x=b$," Computer Methods in Applied Mechanics and Engineering, vol. 195, no. 44-47, pp. 6560-6576, 2006.

[9] C. F. Li, S. Adhikari, S. Cen, Y. T. Feng, and D. R. J. Owen, "A joint diagonalisation approach for linear stochastic systems," Computers and Structures, vol. 88, no. 19-20, pp. 1137-1148, 2010.

[10] F. Wang, C. Li, J. Feng, S. Cen, and D. R. J. Owen, "A novel joint diagonalization approach for linear stochastic systems and reliability analysis," Engineering Computations, vol. 29, no. 2, pp. 221-244, 2012.

[11] W. K. Liu, T. Belytschko, and A. Mani, "Random field finite elements," International Journal for Numerical Methods in Engineering, vol. 23, no. 10, pp. 1831-1845, 1986.

[12] R. G. Ghanem and R. M. Kruger, "Numerical solution of spectral stochastic finite element systems," Computer Methods in Applied Mechanics and Engineering, vol. 129, no. 3, pp. 289-303, 1996.

[13] M. K. Deb, I. M. Babuška, and J. T. Oden, "Solution of stochastic partial differential equations using Galerkin finite element techniques," Computer Methods in Applied Mechanics and Engineering, vol. 190, no. 48, pp. 6359-6372, 2001.

[14] G. Schuëller, "Developments in stochastic structural mechanics," Archive of Applied Mechanics, vol. 75, no. 10-12, pp. 755-773, 2006.

[15] S.-K. Au and J. L. Beck, "Estimation of small failure probabilities in high dimensions by subset simulation," Probabilistic Engineering Mechanics, vol. 16, no. 4, pp. 263-277, 2001.

[16] M. Shinozuka, "Structural response variability," Journal of Engineering Mechanics, vol. 113, no. 6, pp. 825-842, 1987.

[17] S. K. Au and J. L. Beck, "Subset simulation and its application to seismic risk based on dynamic analysis," Journal of Engineering Mechanics, vol. 129, no. 8, pp. 901-917, 2003.

[18] P. S. Koutsourelakis, H. J. Pradlwarter, and G. I. Schuëller, "Reliability of structures in high dimensions-part I: algorithms and applications," Probabilistic Engineering Mechanics, vol. 19, no. 4, pp. 409-417, 2004.

[19] G. I. Schuëller, H. J. Pradlwarter, and P. S. Koutsourelakis, “A critical appraisal of reliability estimation procedures for high dimensions," Probabilistic Engineering Mechanics, vol. 19, no. 4, pp. 463-474, 2004.

[20] J. E. Gentle, Elements of Computational Statistics, Springer, New York, NY, USA, 2002.

[21] Ö. Çavdar, A. Bayraktar, A. Çavdar, and S. Adanur, "Perturbation based stochastic finite element analysis of the structural systems with composite sections under earthquake forces," Steel and Composite Structures, vol. 8, no. 2, pp. 129-144, 2008.

[22] W. K. Liu, T. Belytschko, and A. Mani, "Probabilistic finite elements for nonlinear structural dynamics," Computer Methods in Applied Mechanics and Engineering, vol. 56, no. 1, pp. 61-81, 1986.

[23] E. Vanmarcke and M. Grigoriu, "Stochastic finite element analysis of simple beams," Journal of Engineering Mechanics, vol. 109, no. 5, pp. 1203-1214, 1983.

[24] C. Papadimitriou, L. S. Katafygiotis, and J. L. Beck, "Approximate analysis of response variability of uncertain linear systems," Probabilistic Engineering Mechanics, vol. 10, no. 4, pp. 251264, 1995.
[25] H. G. Matthies, C. E. Brenner, C. G. Bucher, and C. G. Soares, "Uncertainties in probabilistic numerical analysis of structures and solids-stochastic finite elements," Structural Safety, vol. 19, no. 3, pp. 283-336, 1997.

[26] F. Yamazaki, M. Shinozuka, and G. Dasgupta, "Neumann expansion for stochastic finite-element analysis," Journal of Engineering Mechanics, vol. 114, no. 8, pp. 1335-1354, 1988.

[27] M. Shinozuka and G. Deodatis, "Response variability of stochastic finite-element systems," Journal of Engineering Mechanics, vol. 114, no. 3, pp. 499-519, 1988.

[28] R. G. Ghanem and P. D. Spanos, "Spectral stochastic finite-element formulation for reliability analysis," Journal of Engineering Mechanics, vol. 117, no. 10, pp. 2351-2372, 1991.

[29] M. F. Pellissetti and R. G. Ghanem, "Iterative solution of systems of linear equations arising in the context of stochastic finite elements," Advances in Engineering Software, vol. 31, no. 8, pp. 607-616, 2000

[30] D. B. Chung, M. A. Gutiérrez, L. L. Graham-Brady, and F.-J. Lingen, "Efficient numerical strategies for spectral stochastic finite element models," International Journal for Numerical Methods in Engineering, vol. 64, no. 10, pp. 1334-1349, 2005.

[31] A. Keese and H. G. Matthies, "Hierarchical parallelisation for the solution of stochastic finite element equations," Computers and Structures, vol. 83, no. 14, pp. 1033-1047, 2005.

[32] A. Nouy, "A generalized spectral decomposition technique to solve a class of linear stochastic partial differential equations," Computer Methods in Applied Mechanics and Engineering, vol. 196, no. 45-48, pp. 4521-4537, 2007.

[33] A. Nouy, "Generalized spectral decomposition method for solving stochastic finite element equations: invariant subspace problem and dedicated algorithms," Computer Methods in Applied Mechanics and Engineering, vol. 197, no. 51-52, pp. 4718-4736, 2008.

[34] C. E. Powell and H. C. Elman, "Block-diagonal preconditioning for spectral stochastic finite-element systems," IMA Journal of Numerical Analysis, vol. 29, no. 2, pp. 350-375, 2009.

[35] D. Ghosh, P. Avery, and C. Farhat, "An FETI-preconditioned conjugate gradient method for large-scale stochastic finite element problems," International Journal for Numerical Methods in Engineering, vol. 80, no. 6-7, pp. 914-931, 2009.

[36] B. Ganapathysubramanian and N. Zabaras, "Sparse grid collocation schemes for stochastic natural convection problems," Journal of Computational Physics, vol. 225, no. 1, pp. 652-685, 2007.

[37] N. Zabaras and B. Ganapathysubramanian, "A scalable framework for the solution of stochastic inverse problems using a sparse grid collocation approach," Journal of Computational Physics, vol. 227, no. 9, pp. 4697-4735, 2008.

[38] B. Ganapathysubramanian and N. Zabaras, "A seamless approach towards stochastic modeling: sparse grid collocation and data driven input models," Finite Elements in Analysis and Design, vol. 44, no. 5, pp. 298-320, 2008.

[39] B. Ganapathysubramanian and N. Zabaras, "Modeling diffusion in random heterogeneous media: data-driven models, stochastic collocation and the variational multiscale method," Journal of Computational Physics, vol. 226, no. 1, pp. 326-353, 2007.

[40] M. D. Webster, M. A. Tatang, and G. J. McRae, Application of the Probabilistic Collocation Method for an Uncertainty Analysis of a Simple Ocean Model, MIT Joint Program on the Science and Policy of Global Change, 1996. 
[41] I. Babuška, F. Nobile, and R. Tempone, "A stochastic collocation method for elliptic partial differential equations with random input data," SIAM Journal on Numerical Analysis, vol. 45, no. 3 , pp. 1005-1034, 2007.

[42] S. Huang, S. Mahadevan, and R. Rebba, "Collocation-based stochastic finite element analysis for random field problems," Probabilistic Engineering Mechanics, vol. 22, no. 2, pp. 194-205, 2007.

[43] I. Babuška, R. Temponet, and G. E. Zouraris, "Galerkin finite element approximations of stochastic elliptic partial differential equations," SIAM Journal on Numerical Analysis, vol. 42, no. 2, pp. 800-825, 2004.

[44] O. P. Le Maitre, O. M. Knio, H. N. Najm, and R. G. Ghanem, "Uncertainty propagation using Wiener-Haar expansions," Journal of Computational Physics, vol. 197, no. 1, pp. 28-57, 2004.

[45] B. J. Debusschere, H. N. Najm, P. P. Pébayt, O. M. Knio, R. G. Ghanem, and O. P. Le Maître, "Numerical challenges in the use of polynomial chaos representations for stochastic processes," SIAM Journal on Scientific Computing, vol. 26, no. 2, pp. 698719, 2005.

[46] T. Crestaux, O. Le Maître, and J.-M. Martinez, "Polynomial chaos expansion for sensitivity analysis," Reliability Engineering and System Safety, vol. 94, no. 7, pp. 1161-1172, 2009.

[47] R. V. Field Jr. and M. Grigoriu, "On the accuracy of the polynomial chaos approximation," Probabilistic Engineering Mechanics, vol. 19, no. 1, pp. 65-80, 2004. 


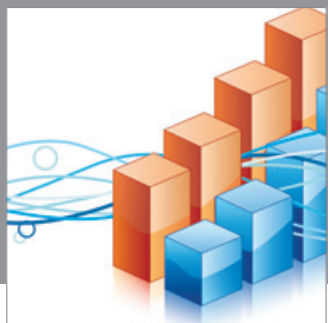

Advances in

Operations Research

mansans

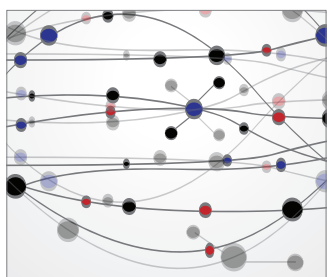

The Scientific World Journal
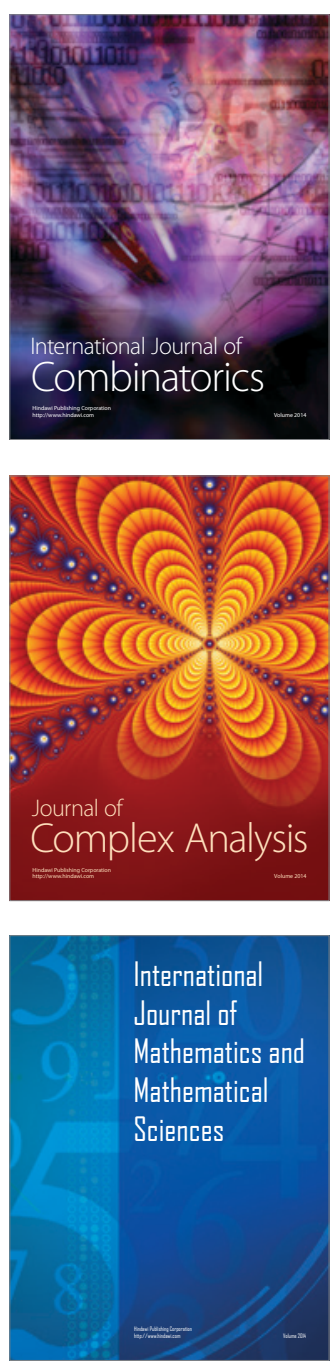
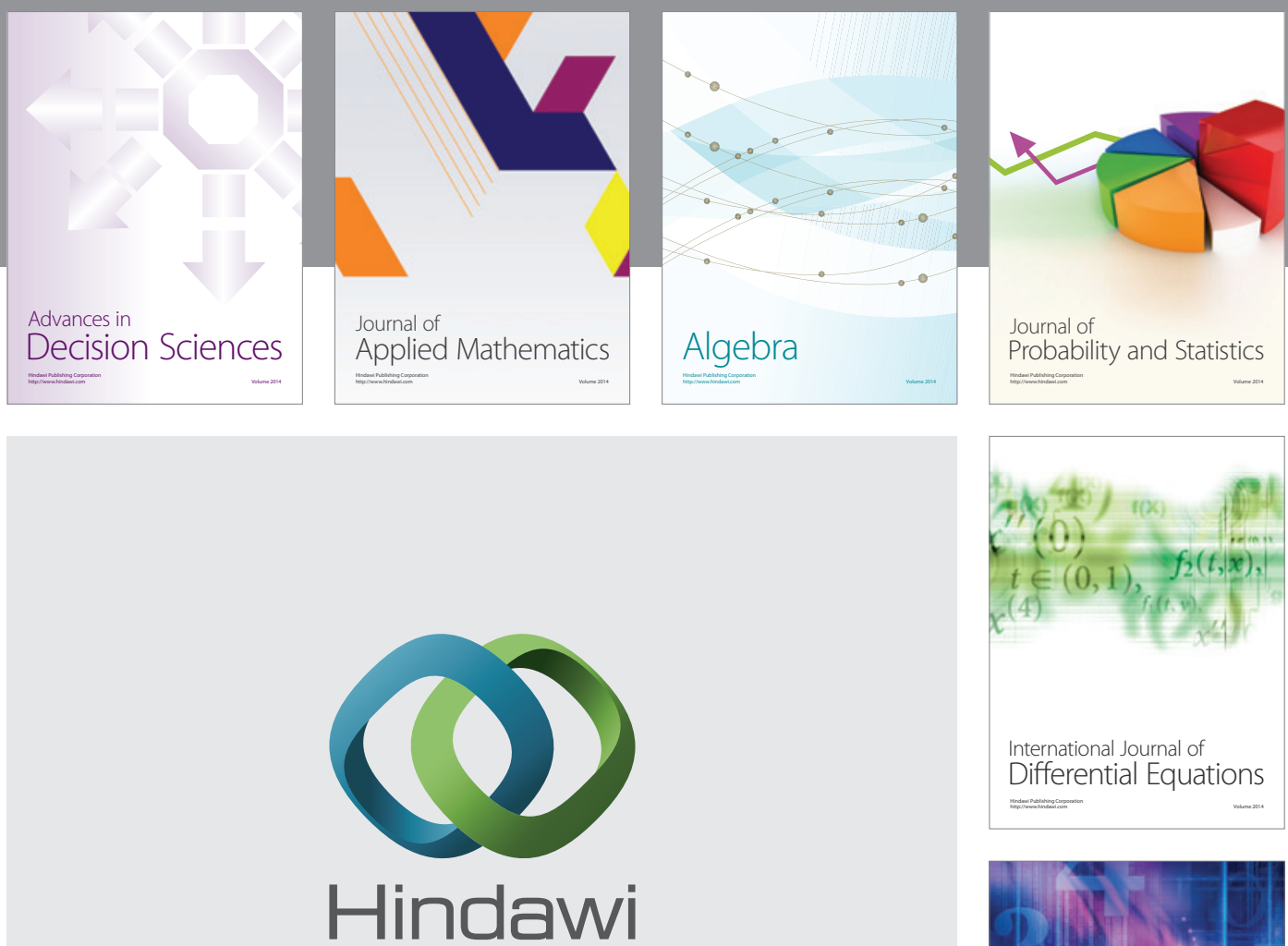

Submit your manuscripts at http://www.hindawi.com
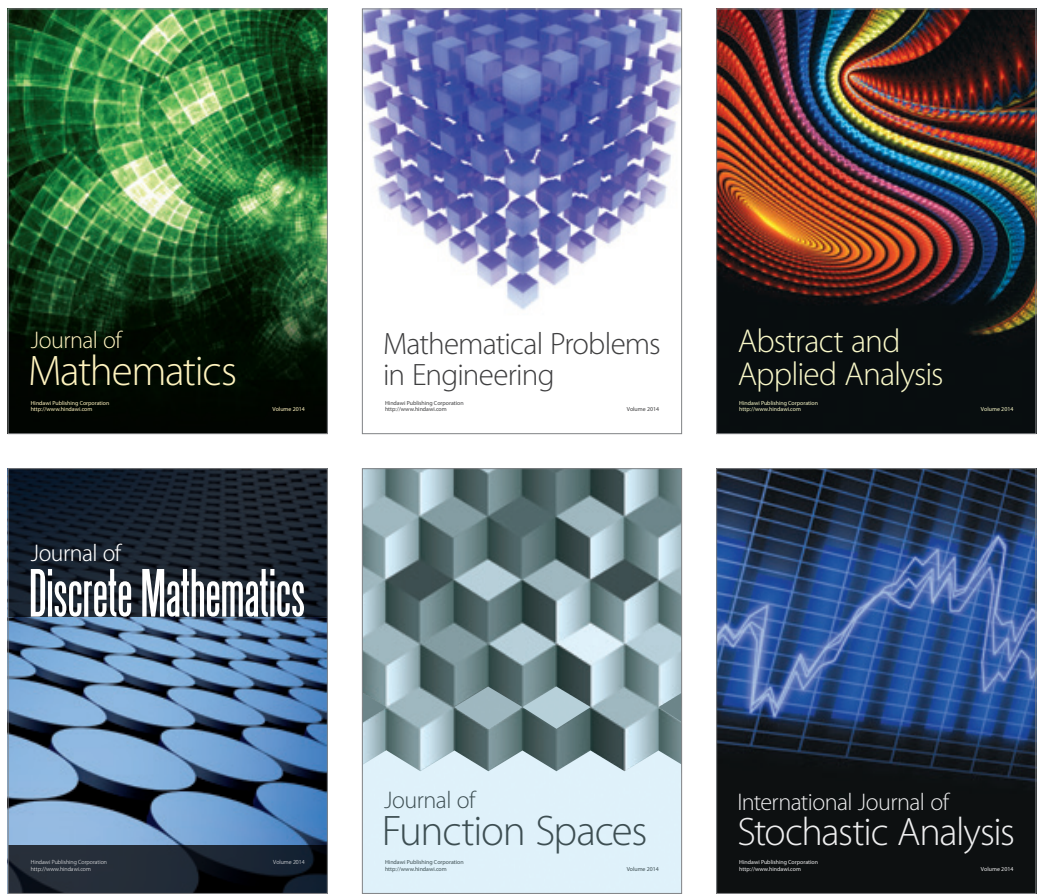

Journal of

Function Spaces

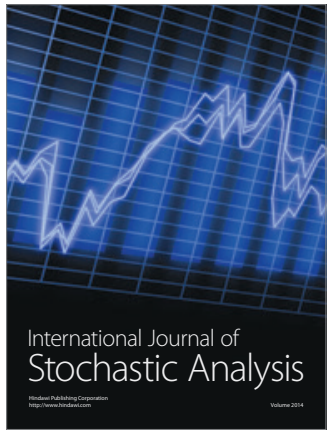

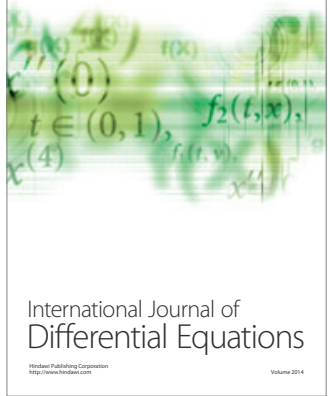
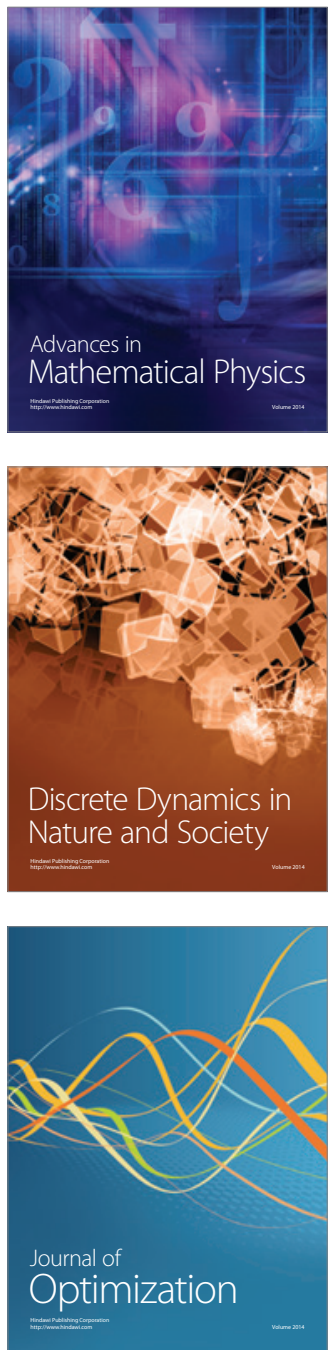